\title{
Pilot study of novel lab methodology and testing of platelet function in adolescent women with heavy menstrual bleeding
}

\author{
Anne D. Rocheleau ${ }^{1}$, Ayesha Khader ${ }^{1}$, Anh T.P. Ngo ${ }^{1}$, Colin Boehnlein ${ }^{2}$, Cara McDavitt ${ }^{2}$, Susan Lattimore ${ }^{2}$, Michael Recht ${ }^{2}$, \\ Owen J.T. McCarty ${ }^{1}$ and Kristina M. Haley ${ }^{2}$
}

BACKGROUND: Approximately $40 \%$ of adolescent women experience heavy menstrual bleeding (HMB), and $10-62 \%$ of them have an underlying bleeding disorder (BD). Diagnosing a $B D$ remains challenging because of limitations of available clinical platelet function assays. The aim of this study was to characterize platelet function in a population of adolescent women with HMB using small-volume whole-blood assays.

METHODS: Anticoagulated whole blood was used to assess platelet GPIIbllla activation, a-granule secretion, and aggregation in response to multiple agonists. Platelet adhesion on collagen or von Willebrand Factor (WWF) under static and shear flow was also assessed.

RESULTS: Fifteen participants with HMB were included in the study, of which eight were diagnosed with a clinically identifiable BD. Platelet activation was blunted in response to calcium ionophore in participants without a BD diagnosis compared with that in all other participants. Impaired GPIIbIlla activation was observed in response to all GPCR agonists, except adenosine diphosphate (ADP), in participants with qualitative platelet disorders. Our assays detected platelet aggregation in the majority of participants with a BD in response to ADP, collagen-related peptide (CRP), thrombin receptor activator 6 (TRAP-6), or U46619. Platelet adhesion and aggregation on collagen and WWF was decreased for participants with WWD.

CONCLUSION: Participants with and without BD exhibited aberrant platelet function in several assays in response to select agonists.

$\mathbf{H}$ eavy menstrual bleeding (HMB), defined as menstrual bleeding lasting longer than 7 days, resulting in blood loss of $>80 \mathrm{ml}$ per menstrual cycle, requiring pads or tampons to be changed hourly, or passage of clots greater than 1 inch in diameter, is the most common symptom of an inherited bleeding disorder (BD) in women (1). HMB frequently results in lost days of school, impaired quality of life, iron deficiency, and increased need for medical and surgical interventions $(1,2)$. Approximately $40 \%$ of adolescent women experience HMB (3), and $10-62 \%$ of adolescent women have a BD (4-8) that causes or contributes to HMB. The true prevalence of $\mathrm{BD}$ in adolescent women with $\mathrm{HMB}$ remains ill-defined, owing to challenges around the definition of normal and abnormal menses, stigma surrounding open discussion of menstrual symptoms, the compounding effect of anovulatory cycles on $\mathrm{HMB}$, lack of awareness of $\mathrm{BD}$ as an etiology for $\mathrm{HMB}$, and challenges associated with clinically available hemostatic testing.

Historically, von Willebrand Disease (VWD) was identified as the most common $\mathrm{BD}$ in females $(1,7)$. However, recent studies of the adolescent population indicate that qualitative platelet disorders (QPD), such as dense granule deficiency or platelet secretion defects, are more common $(4,6,9)$. In a retrospective review of 105 adolescents referred to as an adolescent hematology clinic for evaluation of $\mathrm{HMB}, 36 \%$ were diagnosed with a platelet storage pool disorder using electron microscopy, where abnormal was defined as having fewer than or equal to 3.68 dense granules per platelet (4). In another retrospective review of 131 adolescent women with $\mathrm{HMB}, 11 \%$ were diagnosed with a platelet disorder by platelet aggregation, where abnormal was defined as atypical aggregation response to more than two agonists (8). Electron microscopy is not widely available, and reference ranges are not generalizable between centers (5). In addition, platelet aggregation testing is technologically challenging, requires large volumes of blood, and is not widely available (10-16). The PFA-100 was developed as a screening test for platelet dysfunction, with the advantages of employing whole blood and being technically and logistically more feasible than platelet aggregations. However, it does not reliably identify non-severe platelet abnormalities and is highly sensitive to pre-analytical and analytical variables (17). In a population of women with HMB, Philipp et al. found that the PFA-100 had a low negative predictive value and low sensitivity for platelet dysfunction, and concluded that a normal PFA-100 is not adequate for excluding platelet dysfunction in women with HMB (18). Currently available clinical platelet function assays have significant limitations, including difficulties interpreting abnormalities that do not follow a classic abnormal pattern,

\footnotetext{
${ }^{1}$ Department of Biomedical Engineering, Oregon Health and Science University, Portland, Oregon; ${ }^{2}$ Department of Pediatrics, The Hemophilia Center, Oregon Health and Science University, Portland, Oregon. Correspondence: Kristina M. Haley (haley@ohsu.edu)

Received 26 July 2017; accepted 29 October 2017; advance online publication 3 January 2018. doi:10.1038/pr.2017.298
} 


\section{Articles | Rocheleau et al.}

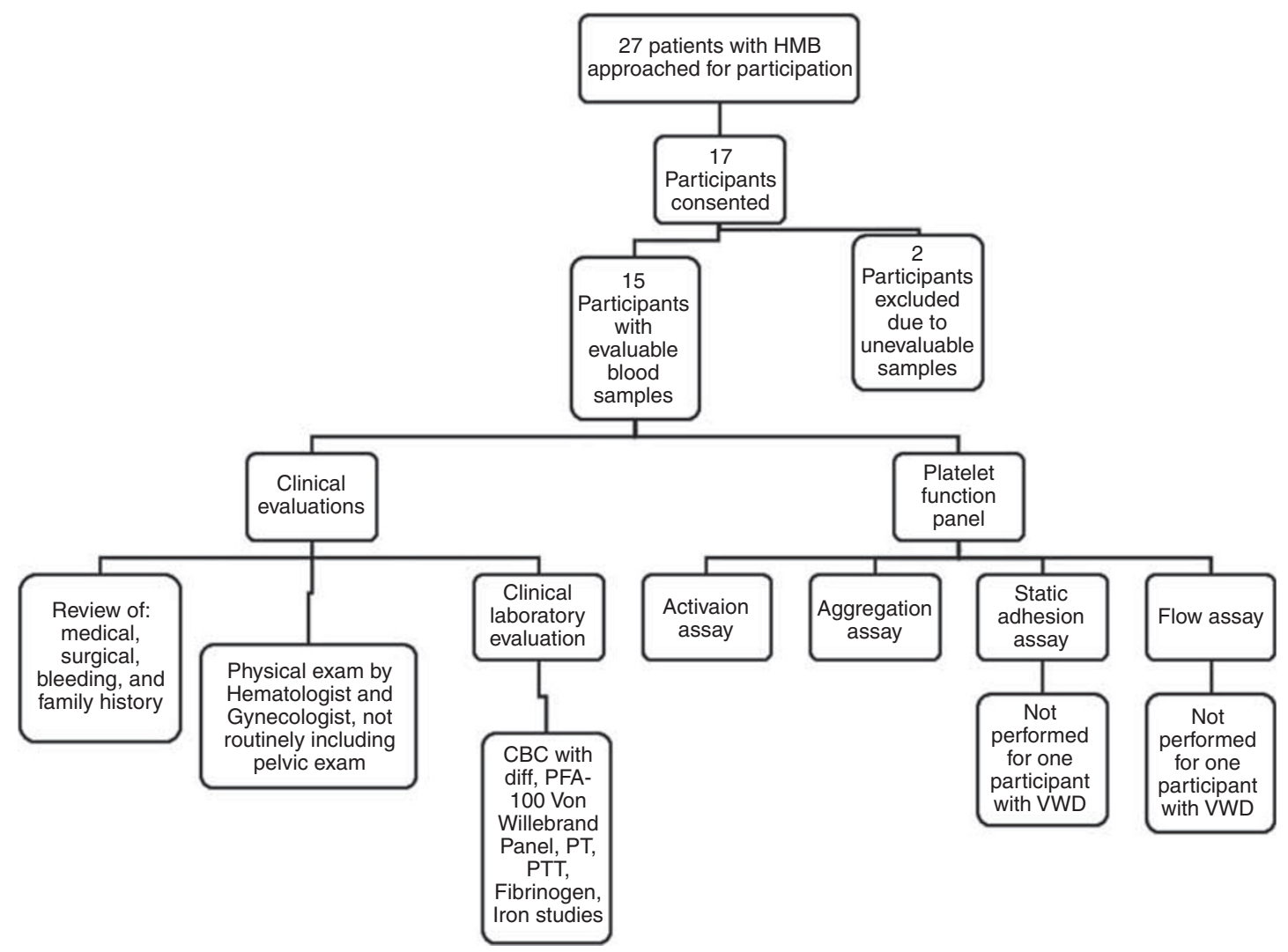

Figure 1. Methodology for study cohort including recruitment, consent, clinical testing and bleeding surveys, and assay function panel.

difficulties in differentiating mild platelet disorders from normal variances, lack of incorporation of the rest of the hemostatic system in the assessment of platelet function, and availability and logistics of testing. In a population of females with mucocutaneous bleeding symptoms, specifically $\mathrm{HMB}$, accurately and efficiently evaluating platelet function is a critical step in the diagnostic pathway.

Our laboratory developed four novel small-volume, wholeblood assays to evaluate platelet function and overcome the deficiencies of clinically available platelet function testing and first employed them to compare neonatal to adult platelet function (19). The four assays assess platelet glycoprotein (GP)IIbIIIa activation, platelet $\alpha$-granule secretion, platelet aggregation, platelet adhesion and spreading on collagen or von Willebrand factor (VWF), and thrombus formation on collagen or VWF under shear flow. These assays have several advantages: the small blood volume (less than $500 \mu \mathrm{l}$ total) allows for easier procurement, whole blood is a physiologically relevant environment that accounts for platelet interactions with other blood elements, each step of platelet function is assessed, and our panel examines the effect of shear force on platelet interactions, which is not currently tested by clinical assays. Given the ability of these assays to robustly evaluate platelet function and data suggesting a higher prevalence than previously reported of platelet dysfunction in women with $\mathrm{HMB}$, we set out to characterize platelet function in adolescent women with HMB in this exploratory study employing our panel of novel assays.

\section{METHODS}

\section{Participant Selection}

This was a single institution pilot study. Adolescent females, aged 12-18 years, referred for evaluation of HMB and seen at the Spots, Dots, and Clots Young Women's Hematology Clinic at Oregon Health and Science University were approached for participation over a 14-month time period. Participants were approached for participation at the end of their clinical visit and before procurement of their clinical blood sample. The study was approved by the Institutional Review Board and written informed consent/assent was obtained from each participant and/or parent/guardian. The study methodology is described in Figure 1.

\section{Clinical Testing and Diagnosis}

As part of the clinical standard of care, complete medical, surgical, family, bleeding, and menstrual histories were obtained and recorded. A bleeding score was assigned using the International Society on Thrombosis and Haemostasis Bleeding Assessment Tool (ISTH-BAT) (20). A score of 3 or higher was considered abnormal for patients aged 12-17 and a score of 6 or higher was considered abnormal for patients aged 18 years (21). In a prospective evaluation of the ISTH-BAT, a score greater than 3 had a $100 \%$ sensitivity but only $2.4 \%$ specificity for $\mathrm{BD}$ diagnosis, whereas a score greater than 5 had a lower sensitivity at $30 \%$ but higher specificity of $88 \%$ (22). The ISTH-BAT score was not used as a diagnostic tool in this study but rather as a tool to characterize the participants' bleeding phenotype. Menstrual bleeding was further assessed using the Philipps tool. The Philipps tool contains eight questions in four categories and is considered positive if there is an affirmative response in any one of 
the four categories (6). All participants underwent a physical examination, and a Beighton score was assigned to assess for joint hypermobility. Participants were evaluated by a gynecologist at the time of their initial consultation as part of local standard of care. All participants underwent institutional standard of care laboratory investigations at their initial consultation including: complete blood count with differential and review of peripheral smear, PFA-100, PTT, PT, fibrinogen, thrombin time, factor VIII activity, VWF activity, VWF antigen, ferritin, total iron-binding capacity, and serum iron (Supplementary Table S1 online), requiring obtainment of up to $40 \mathrm{ml}$ of blood from each participant. Platelet aggregations, flow cytometry quantification of platelet GPIb and GPIIbIIIa surface markers, and thromboelastography were performed at the discretion of the treating provider to identify any platelet-related abnormalities. Diagnosis was assigned by the treatment team and was based on the combination of clinical history, physical examination, and clinical laboratory results. The diagnosis of VWD was assigned according to NHLBI criteria of VWF:Ag and/or VWF:RCo <30 IU/dl and low VWF was assigned if VWF:Ag and/or VWF:RCO was 30-50 IU/dl. These two diagnoses were grouped together as VWD if the participant had no other BD diagnosis. The diagnosis of QPD was based on abnormal platelet aggregation testing except for the participant with concomitant thrombocytopenia. In this participant, platelet-mapping thromboelastography was pursued because of a significant long-standing clinical bleeding history and an upcoming extensive surgical procedure that required hemostatic management. The platelet-mapping thromboelastography demonstrated nearabsent response to ADP, which was not seen on her platelet aggregations. The diagnosis of hypermobile Ehlers Danlos Syndrome (EDS) was based on a Beighton Score of greater than 4. Bleeding disorder, not otherwise specified (NOS), was assigned as a diagnosis to those patients with significant clinical bleeding, including a positive ISTH-BAT score but a negative laboratory work up at the discretion of the treatment provider. If all laboratory testing procedures were normal and the treatment provider's clinical suspicion was low based on the clinical history, physical examination, and ISTH-BAT score, no BD diagnosis was assigned.

\section{Assay Panel}

Citrated venous whole blood collected from each participant was used to quantify PAC-1 monoclonal antibody (mAb) binding and P-selectin expression, platelet-platelet aggregation, static adhesion on collagen and VWF, and adhesion and thrombus formation under low and high shear flow as previously described (19). For the clinical investigations, up to $25 \mathrm{ml}$ of peripheral venous blood was obtained; the experimental assay panel used less than $500 \mu \mathrm{l}$ in all. The activation, aggregation, and static adhesion assays were performed with the following seven agonists: $100 \mu \mathrm{M}$ thrombin receptor activator 6 (TRAP-6; Tocris Bioscience, Bristol, UK); $100 \mu \mathrm{M}$ adenosine diphosphate (ADP; Sigma-Aldrich, St Louis, MO); $100 \mu \mathrm{M}$ thromboxane $\mathrm{A}_{2}$ receptor agonist (U46619; Tocris Bioscience); $100 \mu \mathrm{M}$ epinephrine (Chrono-Log, Havertown, PA); $100 \mu \mathrm{g} / \mathrm{ml}$ collagen-related peptide (CRP; R. Farndale, Cambridge University, Cambridge, UK); $100 \mu \mathrm{M}$ ristocetin (Chrono-Log); and $10 \mathrm{mg} / \mathrm{ml}$ calcium ionophore (Invitrogen, Carlsbad, CA). All antibodies were from BD Biosciences (San Jose, CA), with the exception of eFluor450-CD31 (ThermoFisher, Waltham, MA); FITC-CD31 (ThermoFisher); and FITC-CD62 (OriGene Technologies, Rockville, MD). Other material sources include bovine serum albumin(Sigma-Aldrich); human VWF (Haematologic Technologies, Essex Junction, VT); fibrillary equine type I collagen (Chrono-Log); and cytofix (BD Biosciences).

\section{Activation Assay}

In response to agonist stimulation, the platelet GPIIbIIIa becomes activated, allowing for the formation of platelet aggregates through its binding of fibrinogen. GPIIbIIIa activation can be quantified using the monoclonal antibody PAC-1. In addition, upon agonist stimulation, the activated platelet releases its $\alpha$-granule contents.
P-selectin expression serves as a marker of $\alpha$-granule secretion. Fluorescence-activated cell sorting analysis was employed to quantify the percentage of PAC-1 and P-selectin (CD62P) mAb-positive platelets for each study participant in response to the agonist series. Whole blood was incubated with each agonist and with APC-CD62P $\mathrm{mAb}$ or FITC-PAC-1 mAb for $20 \mathrm{~min}$ at room temperature, followed by fixation and analysis using fluorescence-activated cell sorting as previously described (19).

\section{Aggregation Assay}

The ultimate result of platelet activation is activation of the GPIIbIIIa to mediate platelet aggregation through the binding of fibrinogen. Platelet-platelet bridges form and ultimately result in the generation of an aggregate. In the aggregation assay, whole blood was centrifuged at $1,000 \mathrm{~g}$ for $5 \mathrm{~min}$ before incubating the pellet with CD45-APC and CD31-eFluor450 or CD31-FITC mAb. Removed supernatant was recombined with the pellet and agonist was added, and then incubated at $37^{\circ} \mathrm{C}$ at 1,000 r.p.m. shaking to induce aggregation in a time-dependent manner. Aliquots were removed at time intervals from the time of agonist addition, and double-positive events (CD31-eFluor450+CD31-FITC) were measured using fluorescence-activated cell sorting. Increasing double-positive events indicated increased platelet aggregation, as previously described (19).

\section{Static Adhesion Assay}

Upon endothelial injury and subsequent exposure of extracellular matrix proteins such as collagen or VWF, platelets adhere to the injured surface in order to mediate primary hemostasis. In this assay, platelet adhesion to collagen and VWF was assessed following exposure of platelets to these surfaces. As previously described (13), 5 -mm-diameter circles were created on glass coverslips using a peroxidase-antiperoxidase pen (Invitrogen) before coating with VWF or collagen and blocking with bovine serum albumin. Citrated whole blood was incubated with each agonist, followed by adhesion and spreading on the substrates, and was stained with PE-CD41, FITCCD62P mAb, and 4',6-diamidino-2-phenylindole (DAPI) in 4\% paraformaldehyde. Images were taken at $\times 63$ using a Zeiss Imager M2 microscope using Slidebook software (Intelligent Imaging Innovations, Denver, CO). The average size of adhered platelets for each condition was quantified with Image $1.50 \mathrm{i}$ software (National Institutes of Health). Adhered platelets or aggregates captured in differential interference contrast (DIC) images were circled using the freehand selection tool in ImageJ. Measurement was set to include area under the Analyze tab, and the area in pixels squared was measured. The area in pixels squared was then transformed into $\mu \mathrm{m}^{2}$ by multiplying the pixel-squared values by 0.046656 , a value defined by our imaging software Slidebook.

\section{Flow Assay}

Hemodynamic forces affect platelet function and platelet interactions with other blood components such as VWF. In order to investigate the effect of blood flow on platelet function, microfluidic parallelplate flow chambers (Ibidi u-Slide $\mathrm{VI}^{0.1}$, Munich, Germany) were coated with VWF or collagen and then blocked with bovine serum albumin. Citrated blood was perfused through the channels at shear rates of 200 or $1,500 \mathrm{~s}^{-1}$; the channels were then stained with PE $\mathrm{CD} 41$ and FITC-CD62P mAb, and fixed with DAPI in $4 \%$ paraformaldehyde. Slides were imaged and analyzed at $\times 40$ in the same manner as described above for the static adhesion assay. The average size of adhered platelet aggregates was quantified with Image J as in the static adhesion assay.

\section{RESULTS}

Twenty-seven patients were approached for participation in this study over the course of 14 months. Seventeen consented to participate; however, two of the participants did not undergo testing with the novel platelet assays because of 
sample acquisition difficulties. The results herein present the data for the 14 participants with complete data and 1 participant with data for two out of four assays. The median age for the participants was 15 years (range: 13-18 years). Table 1 lists demographic information, diagnostic results, and bleeding tool information. Eight participants were diagnosed with a clinically identifiable BD, including VWD (2); QPD (3); hypermobile EDS (2); and BD, NOS (1). Seven participants were diagnosed with no clinically identifiable $\mathrm{BD}$. Ten of the fifteen participants had a secondary diagnosis of iron deficiency as determined by serum ferritin measurements. Iron deficiency was defined as ferritin $<12 \mathrm{ng} / \mathrm{ml}$ for participants $<18$ years of age and as ferritin $<50 \mathrm{ng} / \mathrm{ml}$ for participants aged 18 years. The Philipps Screening Tool was positive in 13 participants. Eight of the participants under the age of 18 had an abnormal BAT score $(\geq 3)$, with four of those eight diagnosed with a BD. For the participants with a BAT score $\geq 3$ and not diagnosed with a clinically identifiable $\mathrm{BD}$, one (participant 1) remains lost to follow-up to determine whether HMB symptoms are improved, two (participants 4 and 15) were thought to have other gynecological issues contributing to the high BAT score that were felt to be independent of a $\mathrm{BD}$ by the treating

Table 1. Study cohort characteristics including demographic information, diagnoses, and bleeding survey resultsBAT, Bleeding Assessment Tool; EDS, Ehlers Danlos Syndrome; NOS, not otherwise specified; QPD, qualitative platelet disorder; VWD, von Willebrand disease.

\begin{tabular}{|c|c|c|c|c|}
\hline Participant & $\begin{array}{c}\text { Age } \\
\text { (years) }\end{array}$ & Bleeding disorder diagnosis & Phillips & BAT \\
\hline 1 & 14 & $\begin{array}{l}\text { No clinically identifiable } \\
\text { bleeding disorder; lost to } \\
\text { follow-up }\end{array}$ & + & 3 \\
\hline 2 & 16 & Hypermobile EDS & + & 2 \\
\hline 3 & 18 & Bleeding disorder, NOS & - & 5 \\
\hline 4 & 15 & $\begin{array}{l}\text { No clinically identifiable } \\
\text { bleeding disorder }\end{array}$ & + & 5 \\
\hline 5 & 17 & QPD and thrombocytopenia & - & 3 \\
\hline 6 & 17 & QPD & + & 7 \\
\hline 7 & 14 & Hypermobile EDS & + & 1 \\
\hline 8 & 15 & $\begin{array}{l}\text { No clinically identifiable } \\
\text { bleeding disorder }\end{array}$ & + & 1 \\
\hline 9 & 15 & Type 1 VWD & + & 1 \\
\hline 10 & 17 & Low VWF & + & 4 \\
\hline 11 & 17 & $\begin{array}{l}\text { No clinically identifiable } \\
\text { bleeding disorder }\end{array}$ & + & 4 \\
\hline 12 & 13 & $\begin{array}{l}\text { No clinically identifiable } \\
\text { bleeding disorder }\end{array}$ & + & 2 \\
\hline 13 & 14 & $\begin{array}{l}\text { No clinically identifiable } \\
\text { bleeding disorder }\end{array}$ & + & 2 \\
\hline 14 & 16 & QPD & + & 4 \\
\hline 15 & 14 & $\begin{array}{l}\text { No clinically identifiable } \\
\text { bleeding disorder }\end{array}$ & + & 3 \\
\hline
\end{tabular}

physician, and one (participant 11) had a traumatic brain injury with associated bleeding, which resulted in a higher BAT score.

\section{Platelet Activation and Secretion}

PAC-1 mAb binding to platelets indicates activation of the platelet integrin GPIIbIIIa, and P-selectin mAb binding indicates platelet alpha-granule secretion. The percentage of platelets that stained positive for PAC-1 mAb and P-selectin $\mathrm{mAb}$ binding in response to platelet agonists was analyzed using fluorescence-activated cell sorting (Figure 2). Platelets were stimulated with the G-protein-coupled receptor (GPCR) agonists (ADP, epinephrine, U46619, TRAP-6), the VWFreceptor GPIb agonist (ristocetin), the collagen-receptor GPVI agonist (CRP), or calcium ionophore. Our results show that ADP, U46619, TRAP-6, and CRP induced GPIIbIIIa activation and P-selectin exposure in participants with no clinically identifiable BD, Type 1 VWD, hypermobile EDS, or $\mathrm{BD}$, NOS. In contrast, ADP and CRP stimulated GPIIbIIIa activation and P-selectin exposure in participants with QPD, but GPIIbIIIa activation in response to U46619 and TRAP-6, as well as to epinephrine and ristocetin, was blunted. Interestingly, calcium ionophore failed to induce either GPIIbIIIa activation or P-selectin exposure in platelets from participants with no clinically identifiable $\mathrm{BD}$ and with $\mathrm{BD}, \mathrm{NOS}$, despite eliciting a response for both markers in all other diagnoses. The response to ristocetin was impaired in all conditions for both GPIIbIIIa activation and P-selectin expression.

\section{Platelet Aggregation}

The degree of platelet aggregation was quantified over time in response to select agonists, normalized to baseline (time 0) (Figure 3). Our data show that participants with either Type 1 VWD or no clinically identifiable BD exhibited blunted aggregation in response to the agonists ADP, CRP, TRAP-6, and U46619 as compared with participants with QPD, hypermobile EDS, or BD, NOS diagnoses.

\section{Platelet Adhesion Under Static Conditions}

We quantified the degree of surface area coverage comprising adherent platelets on collagen or VWF in the presence of vehicle or select agonists (Figure 4). This assay was not carried out for participant 10 because of sampling error. Overall, an increase in platelet surface area coverage was observed on VWF surfaces as compared with collagen surfaces. Similar trends of increased surface area coverage in response to GPCR- or GPVI agonists were observed for all participants, except for the participant with QPD and thrombocytopenia in whom there was no response to CRP on the VWF surface.

Platelet Adhesion and Thrombus Formation Under Shear Flow We quantified the degree of platelet adhesion and aggregation on collagen and VWF under shear flows of 200 and 1,500 s-1 for $30 \mathrm{~s}$; results are reported as the degree of surface area coverage (Figure 5). This assay was not performed for 

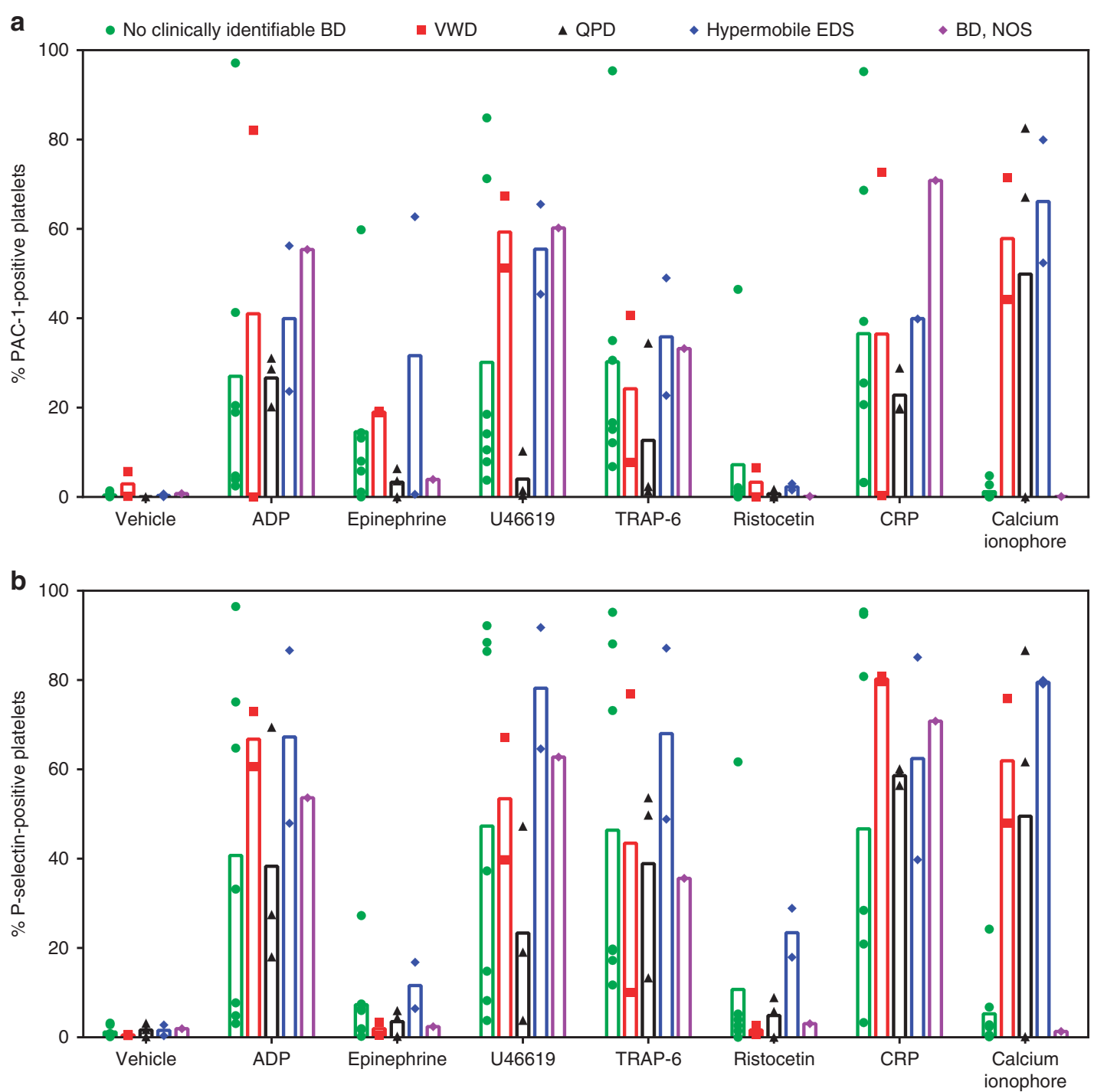

Figure 2. Platelet activation (PAC-1) (a) and secretion (P-selectin) (b) for study cohort. (a) PAC-1 and (b) P-selectin expression for study cohort. Individual participant results are plotted as symbols and the mean averages for each agonist condition per diagnosis are represented by bars. The symbols and bars are color-coded by diagnosis: green indicates no clinically identifiable BD; red indicates VWD; black indicates QPD; blue indicates hypermobile EDS; and purple indicates BD, NOS. Participant 5 has thrombocytopenia in addition to QPD and this is indicated by open symbols. BD, bleeding disorder; EDS, Ehlers Danlos Syndrome; NOS, not otherwise specified; QPD, qualitative platelet disorder; VWD, von Willebrand disease.

participant 10 because of sampling error. Our data show that platelet surface coverage increased on collagen or VWF surfaces in a shear-dependent manner for no clinically identifiable $\mathrm{BD}, \mathrm{QPD}$ and hypermobile EDS or $\mathrm{BD}$, NOS participants. An overall decrease in platelet surface area coverage was observed for participant 5 , who was diagnosed with thrombocytopenia, except for on VWF at $1,500 \mathrm{~s}^{-1}$. Platelet surface area coverage was markedly decreased on all surfaces and at all rates for participant 9 , who was diagnosed with Type 1 VWD.

\section{DISCUSSION}

In this exploratory study, a panel of four whole blood, smallvolume platelet function assays previously used to compare platelet function in healthy neonates to adults (19) was employed to characterize platelet function in a population of 15 adolescent women with HMB. Using standard clinical hemostasis assays or clinical examination, three participants were diagnosed with a QPD, two were diagnosed with VWD, two were diagnosed with hypermobile EDS, one was diagnosed with BD, NOS, and seven were not diagnosed with a clinically identifiable BD. Four of the participants who were not diagnosed with a BD had positive BAT scores and seven had positive Philipps Screening Tools, raising the concern that these patients may have an underlying BD not captured by clinically available assays. The lack of specific diagnoses, as well as the positive BAT scores without reaching a clinical $\mathrm{BD}$ diagnosis, highlights the challenges of the 

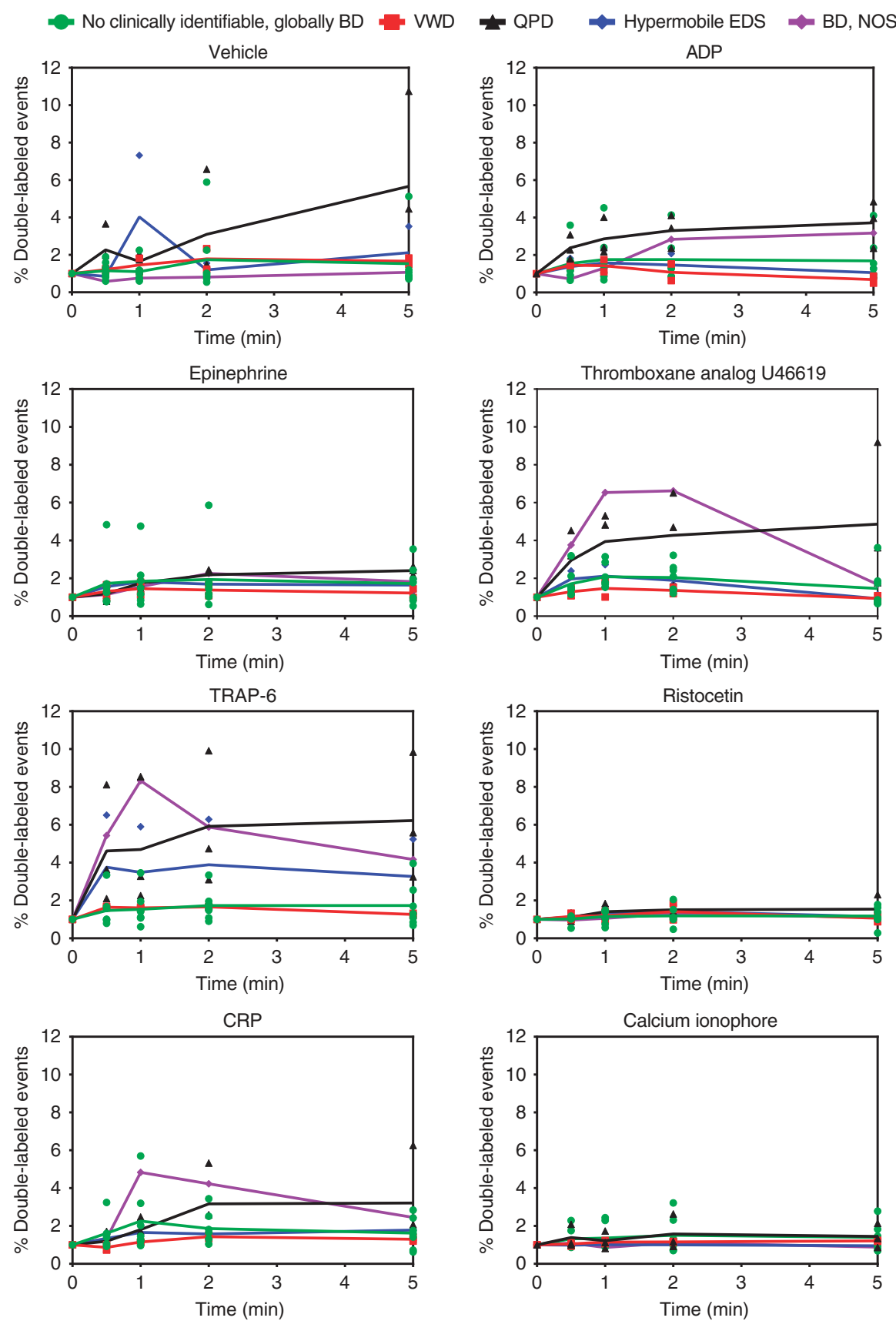

Figure 3. Platelet aggregation for the study cohort. Individual participant results are plotted as symbols and the mean averages for each agonist condition per diagnosis are connected by lines. The symbols and lines are color-coded by diagnosis: green indicates no clinically identifiable BD; red indicates VWD; black indicates QPD; blue indicates hypermobile EDS; and purple indicates BD, NOS. BD, bleeding disorder; EDS, Ehlers Danlos Syndrome; NOS, not otherwise specified; QPD, qualitative platelet disorder; VWD, von Willebrand disease.

current standard laboratory evaluation of patients with HMB or other mucocutaneous bleeding symptoms and represents unmet clinical needs for better diagnosis. Our small-volume (less than $500 \mu \mathrm{l}$ of blood) platelet function assays were added to the institutional standard hemostasis testing to explore platelet function in this population more fully, as well as to investigate the effectiveness of this assay panel as a potential novel diagnostic tool. Overall, we observed heterogeneity in markers of GPIIbIIIa activation and P-selectin exposure for patients with a BD. Yet, except for the case of the VWD patient, a deficiency in platelet activation did not manifest as a functional decrease in the hemostatic function of platelets in initiating thrombus formation under flow on either collagen or VWF. Thus, these assays hold potential for use as screening tools to identify patients with a platelet dysfunction, but further studies must be carried out to improve the ability to assess the risk of bleeding in these patients. 

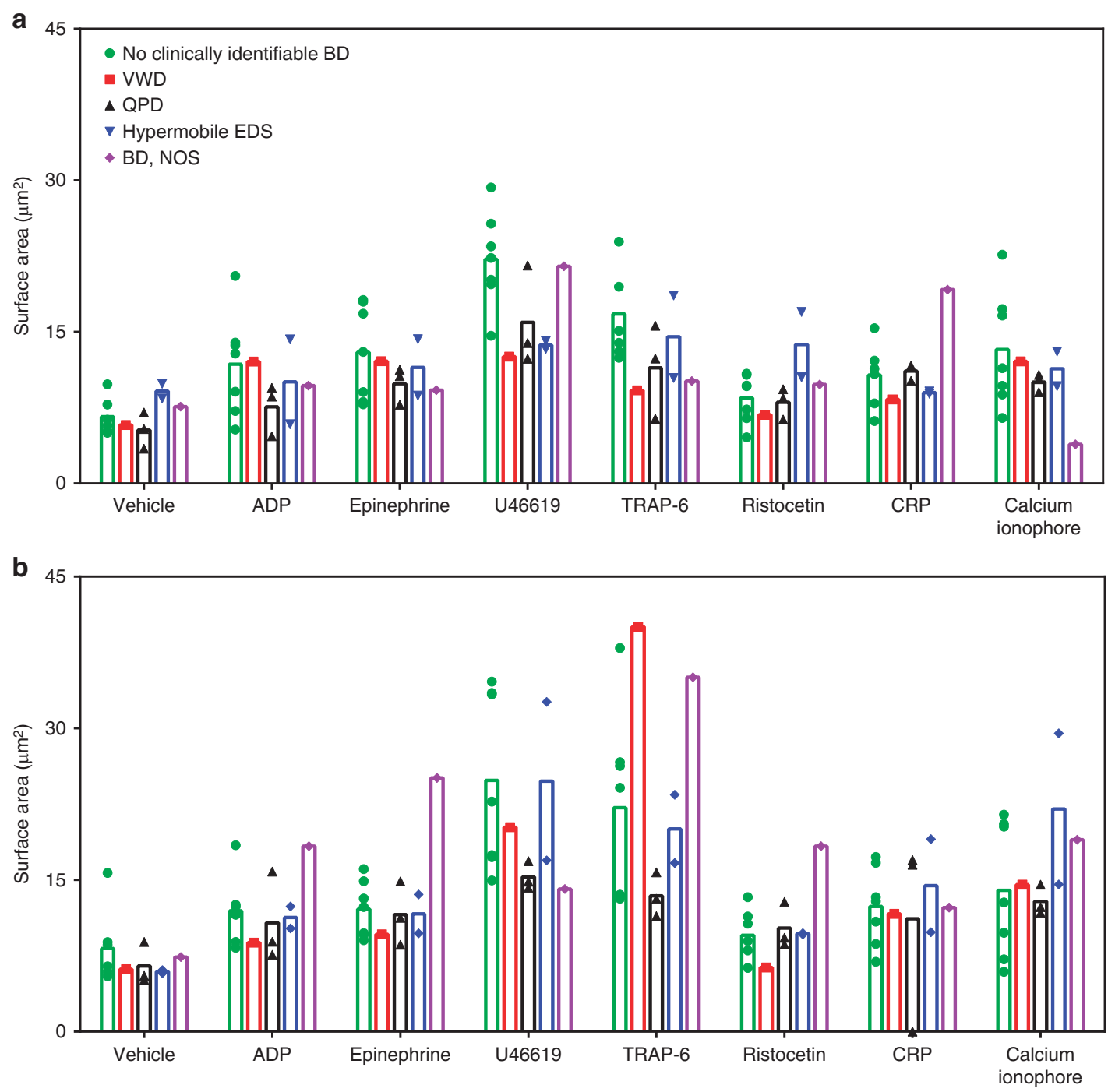

Figure 4. Platelet adhesion and spreading for study cohort on (a) collagen and (b) VWF surfaces. Individual participant results are plotted as symbols and the mean averages for each agonist condition per diagnosis are represented by bars. The symbols and bars are color-coded by diagnosis: green indicates no clinically identifiable BD; red indicates VWD; black indicates QPD; blue indicates hypermobile EDS; and purple indicates BD, NOS.

Participant 5 has thrombocytopenia in addition to QPD, and this is indicated by open symbols. This assay was not performed for participant 10. BD, bleeding disorder; EDS, Ehlers Danlos Syndrome; NOS, not otherwise specified; QPD, qualitative platelet disorder; VWD, von Willebrand disease.

GPIIbIIIa activation in response to the GPCR agonists epinephrine, U46619, and TRAP-6, but not ADP, was impaired in participants with QPD. However, P-selectin expression was more similar to other diagnoses and aggregation was more robust for the QPD participants than for the others. Together, these results suggest that GPCR signaling leading to GPIIbIIIa activation is selectively impaired, and that ADP signaling may provide adequate stimulation for aggregation either as a primary agonist or as a co-stimulatory agent. In clinical testing, QPD participants numbered 6 and 14 exhibited impaired platelet aggregation in response to ADP and epinephrine (Supplementary Table S1). In our platelet aggregation assay, we observed mild impairment in response to epinephrine, but not to ADP. This difference could be due to the insensitivity of standard clinical aggregometry to smaller platelet aggregates (23); the variation in agonist concentrations employed; the time between blood collection and assay performance; or the utilization of platelet-rich plasma in the clinical testing vs. whole blood in the smallvolume assays. For the participant with QPD and thrombocytopenia, the adhesion assays were sensitive enough to ascertain that there was reduced platelet adhesion under static and flow conditions, commensurate with less static adhesion observed at lower washed platelet concentrations (13). Notably, at $1,500 \mathrm{~s}^{-1}$ on the VWF surface, platelet adhesion for the QPD/thrombocytopenic participant was higher than that for any other participant, suggesting that higher shear rates may overcome the effect of thrombocytopenia or platelet dysfunction.

The VWD participant had low platelet surface area coverage in the flow assay compared with other participants, especially at the higher flow rate. Previous work has shown 


\section{Articles | Rocheleau et al.}

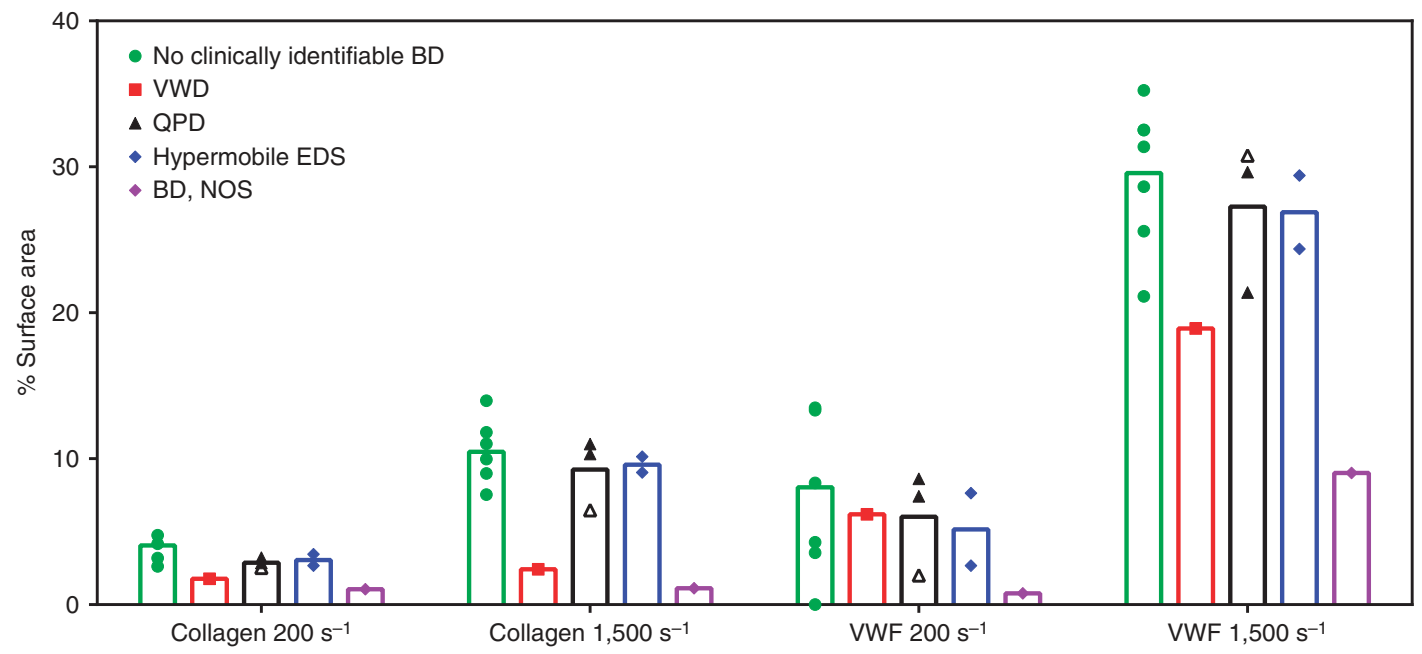

Figure 5. Percentage of flow channel covered with platelets and/or platelet aggregates for each study participant at shear rates of and $1,500 \mathrm{~s}^{-1}$ on VWF and collagen surfaces. The participant symbols are color-coded by diagnosis: green indicates no clinically identifiable BD; red indicates VWD; black indicates QPD; blue indicates hypermobile EDS; and purple indicates BD, NOS. Participant 5 also has a thrombocytopenia diagnosis, and this is indicated by open symbols. This assay was not performed for participant 10. BD, bleeding disorder; EDS, Ehlers Danlos Syndrome; NOS, not otherwise specified; QPD, qualitative platelet disorder; VWD, von Willebrand disease.

that platelets interacting with a VWF surface aggregate independently of activation when soluble VWF is present under a high shear rate [ (16)]. As the VWD participant had low VWF levels, decreased surface area coverage on the VWF surface was anticipated. However, in the activation and static adhesion assays, the responses in the VWD participants were more similar to other participants, suggesting that presence of agonist stimulation overcame the low VWF concentration. The effect of VWF on platelet function may help explain the significant bleeding phenotype heterogeneity exhibited within the Type 1 VWD population. This may highlight a compensatory mechanism through enhanced GPIb-mediated adhesion in the presence of normal or high VWF concentration. Alternatively, in participants with platelet dysfunction who also have low VWF, bleeding phenotype may be more severe. Further work is needed to fully understand the effect of shear forces on platelet function in patients with bleeding symptoms and QPD to determine whether shear forces can compensate for platelet dysfunction or inform treatment decisions.

Interestingly, the participants who were not assigned a clinical $\mathrm{BD}$ had a very minimal response to a calcium ionophore in the activation assay. As this pathway is not specifically evaluated by clinically available assays, it may highlight a novel platelet dysfunction leading to HMB, and further studies are needed to investigate the effect of calcium on platelet signaling in participants with mucocutaneous bleeding. The contributions of hormonal interventions and iron deficiency to our experimental results were not assessed. The data were evaluated for similarities in assay results across BAT scores, Pictorial Bleeding Assessment Chart (PBAC), BMI, or age (Supplementary Table S1), and no patterns emerged with regard to platelet function.

This study has several limitations. The small sample size limits the ability to make further conclusions from this study but allows for additional generation of hypotheses around the contribution of platelet dysfunction to HMB. Ten participants had a secondary diagnosis of iron deficiency, and the degree to which this contributed to the experimental results is not clear. In addition, the majority were on some form of hormonal management for their $\mathrm{HMB}$, and it is unknown how these medications contributed to the experimental results. Further investigation is required to establish a baseline range of values for this platelet function assay panel for individuals of all ages.

To summarize, we performed a pilot study of a cohort of 15 adolescent women with HMB employing novel small volume platelet function assays. On the basis of clinical history and standard clinical hemostatic labs, eight were diagnosed with a clinically identifiable $\mathrm{BD}$, including $\mathrm{QPD}$, VWD, hypermobile EDS, or BD, NOS. In our experimental assays, participants with QPD had lower GPIIbIIIa activation in response to all tested GPCR agonists except for ADP. Participants with VWD had lower platelet adhesion and aggregation. In most participants with a $\mathrm{BD}$, there was platelet aggregation after exposure to ADP, CRP, TRAP-6, or U46619. Participants without a clinically $\mathrm{BD}$ diagnosis exhibited a blunted response to calcium ionophore in the platelet activation assay.

$\mathrm{HMB}$ is a common problem for adolescent women and may be the first and/or only sign of a BD. As HMB is a type of mucocutaneous bleeding, it is thought that platelet disorders are a common cause of HMB. Clinically available platelet function assays are limited with regard to availability, reproducibility, and accuracy. We applied a panel of four small-volume-whole-blood assays to a cohort of adolescent women with HMB. The assays detected differences in platelet function among subjects with different diagnoses and demonstrated platelet dysfunction in participants who were not assigned a clinical BD. Further work is needed to better 
characterize calcium signaling as well as the effect of ADP signaling and platelet secretion in depth. In addition, ongoing evaluation of the effect of shear forces on platelet function in participants with mucocutaneous bleeding remains crucial to our understanding of the bleeding phenotype.

\section{SUPPLEMENTARY MATERIAL}

Supplementary material is linked to the online version of the paper at http://www.nature.com/pr

\section{STATEMENT OF FINANCIAL SUPPORT}

This study was supported by program income funds generated by participation in HRSA Award \#H30MC24049, the Doernbecher Children's Hospital Foundation-Friends of Doernbecher Grant, and by grants from the National Institutes of Health (R01HL101972 and R01GM116184 to O.J.T. M.). O.J.T.M. is an American Heart Association Established Investigator (13EIA12630000).

Disclosure: The authors declare no conflict of interest.

\section{REFERENCES}

1. James AH. Women and bleeding disorders. Haemophilia 2010;16:160-7.

2. Konkle BA. Progress toward meeting the needs of adolescent females with bleeding disorders. Haemophilia 2016;22:196-8.

3. Friberg B, Ornö AK, Lindgren A, Lethagen S. Bleeding disorders among young women: a population-based prevalence study. Acta Obstet Gynecol Scand 2006;85 (December 2004): 200-6.

4. Vo KT, Grooms L, Klima J, Holland-Hall C, O’Brien SH. Menstrual bleeding patterns and prevalence of bleeding disorders in a multidisciplinary adolescent haematology clinic. Haemophilia 2013;19:71-5.

5. Zia A, Rajpurkar M. Challenges of diagnosing and managing the adolescent with heavy menstrual bleeding. Thromb Res 2016;143:91-100.

6. Philipp CS, Faiz A, Dowling N, et al. Age and the prevalence of bleeding disorders in women with menorrhagia. Obstet Gynecol 2005;105:61-6.

7. Mikhail S, Kouides P. Von Willebrand disease in the pediatric and adolescent population. J Pediatr Adolesc Gynecol 2010;23 (SUPPL. 6): S3-10.

8. Díaz R, Dietrich JE, Mahoney D, Yee DL, Srivaths LV. Hemostatic abnormalities in young females with heavy menstrual bleeding. J Pediatr Adolesc Gynecol 2014;27:324-9.

9. Seravalli V, Linari S, Peruzzi E, Dei M, Paladino E, Bruni V. Prevalence of hemostatic disorders in adolescents with abnormal uterine bleeding. J Pediatr Adolesc Gynecol 2013;26:285-9.
10. Mezzano D, Quiroga T, Pereira J. The level of laboratory testing required for diagnosis or exclusion of a platelet function disorder using platelet aggregation and secretion assays. Semin Thromb Hemost 2009;35: $242-54$.

11. Jennings LK, Kotha J. The utility of platelet and coagulation testing of antithrombotics: fusing science with patient care. Drug Dev Res 2013;74: 587-93.

12. Dovlatova N. Current status and future prospects for platelet function testing in the diagnosis of inherited bleeding disorders. Br J Haematol 2015;170:150-61.

13. Baker-Groberg SM, Cianchetti FA. Development of a method to quantify platelet adhesion and agreggation under static conditions. Cell Mol Bioeng 2014;4:285-90.

14. Baker-Groberg SM, Phillips KG, Healy LD, et al. Critical behavior of subcellular density organization during neutrophil activation and migration. Cell Mol Bioeng 2015;8:543-2.

15. Aslan JE, Rigg RA, Nowak MS, et al. Lysine acetyltransfer supports platelet function. J Thromb Haemost 2015;13:1908-7.

16. Baker-Groberg SM, Bornstein S, Zilberman-Rudenko J, et al. Effect of ionizing radiation on the physical biology of head and neck squamous cell carcinoma cells. Cell Mol Bioeng 2015;8:517-25.

17. Greaves M, Watson HG. Approach to the diagnosis and management of mild bleeding disorders. J Thromb Haemost 2007;5 (Suppl 1): 167-74.

18. Philipp CS, Miller CH, Faiz A, et al. Screening women with menorrhagia for underlying bleeding disorders: the utility of the platelet function analyser and bleeding time. Haemophilia 2005;11:497-503.

19. Baker-Groberg SM, Lattimore S, Recht M, McCarty OJT, Haley KM. Assessment of neonatal platelet adhesion, activation, and aggregation. J Thromb Haemost 2016;14:815-27.

20. Rodeghiero F, Tosetto A, Abshire T, Arnold DM, Coller B, James P. ISTH/SSC bleeding assessment tool: a standardized questionnaire and a proposal for a new bleeding score for inherited bleeding disorders. J Thromb Haemost 2010;8:2063-5.

21. Elbatarny M, Mollah S, Grabell J, Bae S, Deforest M, Tuttle A. Normal range of bleeding scores for the ISTH-BAT : adult and pediatric data from the merging project. Haemophilia 2014;20:831-5.

22. Jain S, Sarode R, Journeycake JM, Zia A. Prospective evaluation of ISTHBAT as a predictor of bleeding disorder in adolescent girls with heavy menstrual bleeding. Blood 2016;128:1402.

23. Thompson NT, Scrutton MC, Wallis RB. Particle volume changes associated with light transmittance changes in the platelet aggregometer: dependence upon aggregating agent and effectiveness of stimulus. Thromb Res 1986;41:615-26. 